\title{
Concepciones de docentes sobre los estudiantes y sus prácticas pedagógicas ${ }^{1}$
}

\author{
Ana Borgobello \\ orcid.org/0000-0002-2340-8127 \\ Universidad Nacional de Rosario, \\ Argentina \\ borgobello@irice-conicet.gov.ar
}

\section{Mariana Sartori}

orcid.org/000o-0001-6921-5462

Universidad Nacional de Rosario, Argentina

sartori@irice-conicet.gov.ar

\section{Liliana Sanjurjo}

orcid.org/0000-0003-3113-0169

Universidad Nacional de Rosario,

Argentina

sanjurjo@irice-conicet.gov.ar

\section{Resumen}

En Argentina, particularmente en la ciudad de Rosario, la implementación de entornos virtuales en educación a distancia o en combinación con clases presenciales-enseñanza bimodal- es aún incipiente y depende, en la mayoría de los casos, de la voluntad individual o de pequeños colectivos docentes. Teniendo en cuenta el contexto local, se analizaron las narraciones de diez docentes universitarios entrevistados que comenzaban a utilizar un entorno virtual como complemento de sus aulas regulares de clases. Los ejes de este análisis fueron: caracterizaciones de sus estudiantes ideales y habituales, concepciones sobre trabajo colaborativo grupal (TCG) y dificultades, expectativas y temores ante la experiencia por venir. Los docentes describieron de modo enfático diferencias entre sus estudiantes habituales e ideales; usualmente, proponían TCG y valoraban positivamente este tipo de estrategia pedagógica. Si bien la mayor dificultad esperada en el trabajo en la plataforma se relacionó con su

$1 \quad$ Organismos colaboradores: Consejo Nacional de Investigaciones Científicas y Tecnológicas; Universidad Nacional de Rosario; Universidad Abierta Interamericana. Financiamiento de la Agencia Nacional de Promoción Científica y Tecnológica (PICT2013-17).

Recepción: 28/04/2017 | Envío a pares:30/10/2017 | Aceptación por pares: 12/11/2017 | Aprobación: 30/11/2017 
ISSN 0123-1294 | e-ISSN 2027-5358 | Educ.Educ. Vol. 21. No. 1 | Enero-abril de 2018 | pp. 27-48.

Universidad de La Sabana | Facultad de Educación

manejo técnico, los resultados mostraron diversidad en los temores y las dificultades esperados. Como categoría emergente, surgió que una de las mayores preocupaciones de los docentes es la motivación de los estudiantes tanto en sus clases presenciales como para con el uso de Moodle.

\section{Palabras clave}

Educación universitaria; educación a distancia; enseñanza asistida por ordenador; prácticas pedagógicas; Argentina (Fuente: Tesauro de la Unesco). 


\title{
Teachers' Ideas about Students and their Teaching Practices
}

\begin{abstract}
In Argentina, the use of virtual environments in e-learning or in blended learning designs is mostly a question of individual willingness or that of a small group. Considering the local context, we analyzed the narratives of ten university teachers who began to use a virtual environment as a complement to their regular classes. The analysis axes were characterizations of their ideal and regular students, conceptions about group collaborative learning, and expected fears and difficulties with the experience to come. Teachers emphatically described the differences between their usual students and their ideal students. They normally proposed collaborative tasks for their students and positively valued this type of teaching strategy. Nevertheless, the most important expected difficulty was the technical management of the platform and the results showed diversity with respect to their anticipated difficulties. It emerged from the data that one of the main concerns of teachers was the students' motivation in terms of both regular classes and for the future use of Moodle.
\end{abstract}

\section{Keywords}

Higher education; Distance education; Computer assisted instruction; Teaching practice; Argentina (Source: Unesco Thesaurus). 


\section{Concepções de docentes sobre os estudantes e suas práticas pedagógicas}

\section{Resumo}

Na Argentina, especialmente na cidade de Rosario, a implementação de ambientes virtuais no ensino a distância ou em combinação com aulas tradicionais, ainda depende, na maioria dos casos, da vontade de um professor ou de pequenos grupos de professores. Considerando o contexto local, foram analisadas as histórias de dez professores universitários que começaram a usar um ambiente virtual para complementar suas aulas regulares. Os eixos desta análise foram: caracterizações de seus ideais e estudantes comuns, concepções de grupo colaborativo de trabalho e expectativas, medos e dificuldades com a experiência. Assim, os professores descreveram diferenças enfáticas entre estudantes atuais e ideais. Em geral, eles propuseram trabalho colaborativo a seus estudantes e valorizaram positivamente esse tipo de estratégia de ensino. Embora a maior dificuldade esperada em trabalhar com a plataforma esteja relacionada com a parte técnica, os resultados mostraram diversidade de medos e dificuldades esperados. Como uma categoria emergente, verificou-se que uma das principais preocupações dos professores foi a motivação dos alunos em suas aulas e o uso do Moodle.

\section{Palavras-chave}

Educação universitária; ensino a distância; ensino auxiliado por computador; práticas pedagógicas; Argentina (Fonte: Tesauro da Unesco). 


\section{Introducción}

El presente artículo tiene como propósito explorar conceptualizaciones de docentes universitarios en relación con diversos aspectos centrales que afectarían la implementación y el uso de entornos virtuales. Los múltiples ejes de análisis abordados fueron temas cruciales en el uso de plataformas, ya que las concepciones del docente acerca de los estudiantes, de cómo propician las interacciones y de cómo entienden el uso de tal entorno condicionan las prácticas de enseñanza y el diseño tecnopedagógico.

Aquí se analizan resultados preliminares de un proyecto más amplio sobre interacción sociocognitiva en entornos virtuales con diseño multimétodo en el que un grupo de diez docentes participaron voluntariamente de un taller de formación básica en uso de la plataforma Moodle. Los participantes pertenecían a una universidad pública y otra privada de la ciudad de Rosario, Argentina, todos eran jefes de trabajos prácticos y fueron convocados para un taller de diseño de cursos en Moodle para luego construir sus propias aulas virtuales. Previo al taller se realizaron entrevistas semiestructuradas donde se abordaron diversos ejes temáticos. Después, los docentes diseñaron e implementaron sus cursos con uso de plataforma virtual del modo que consideraron pertinente. Al terminar el curso con sus estudiantes, por un lado, se realizaron entrevistas grupales y se aplicaron cuestionarios a los estudiantes y, por el otro, se hicieron nuevas entrevistas en profundidad a los docentes, a modo de evaluación conjunta de la experiencia. Asimismo, se analizaron los índices de participación de los estudiantes, las modalidades de elaboración del guion (scripting), entre otras observaciones post facto de la interacción sociocognitiva en el propio entorno digital.

Con esta perspectiva de trabajo, se considera a los docentes como coinvestigadores, acorde a los cambios profundos que se produjeron en las últimas décadas en investigación psicoeducativa. Para Coll y Sánchez (2008), por un lado, se comenzó a considerar que ser investigador o profesor pueden ser actividades complementarias y no excluyentes. Esta nueva lógica se gestó como consecuencia de dificultades prácticas que los profesores encontraban para aplicar resultados de investigaciones a sus propias clases. De esta manera, se comenzó a afianzar el concepto de docente-investigador. Por otro lado, el aula pasó a ser contexto de enseñanza y de aprendizaje, construido tanto por docentes como por estudiantes, entendiéndose que la construcción del conocimiento es una actividad situada y que se da en interacción con otros.

En este marco se inscribe el presente artículo, que reúne la narrativa y percepciones de docentes que se iniciaban en el uso de Moodle como espacio virtual de complemento del aula tradicional de clases. Se parte de la consideración de que, en un proyecto cuyo eje es la interacción sociocognitiva, resulta pertinente analizar la narrativa y las percepciones de los docentes, ya que lo que piensan tiene efectos directos en la forma en que estructuran sus clases $y$, por tanto, en la manera como se relacionan contenidos, estudiantes y docentes en prácticas áulicas concretas. Que las tecnologías de la información y la comunicación (TIC) puedan ser utilizadas efectivamente como instrumentos psicológicos, dependerá de cómo se las ubique en el marco de relaciones entre los componentes del triángulo interactivo: contenido (objeto de enseñanza y aprendizaje), actividad educativa del profesor y actividad de aprendizaje de los estudiantes (Bustos y Coll, 2010).

La orientación del escrito se sustenta en una lógica vigotskiana, fundamentalmente heurística, que da lugar a un análisis contextualizado e históricamente situado de las narraciones y percepciones de los docentes. Incluimos conceptos para pensar la educación como objeto de estudio complejo atravesado por lógicas de investigación diversas, en especial en la actualidad, con la incorporación de lo virtual como parte de ese fenómeno. Son ampliamente conocidos los cambios acaecidos en las universidades a partir de la incorporación de sistemas de comunicación indirectos, tales como correos 
electrónicos, dispositivos móviles y plataformas virtuales educativas, entre otros. Estas tecnologías de la información y la comunicación se encuentran al alcance de docentes y estudiantes de nivel superior en forma casi masiva. Sin embargo, su uso pedagógico sistemático es aún restringido, al menos en el ámbito universitario local.

\section{Contexto local y conceptual}

$\mathrm{El}$ contexto en que se centra el presente estudio es la Universidad Nacional de Rosario (UNR), que dispone de un campus virtual con el entorno Moodle accesible a la comunidad universitaria desde 2001 (Copertari, 2010). Si bien la apreciación de una pobre utilización de los espacios virtuales con extrapolación de metodologías propias de los cursos presenciales no es algo exclusivo del contexto en el que se desarrolló la experiencia (Zúñiga y Martínez, 2015), las experiencias locales muestran modestos avances, pero también preocupaciones sobre la implementación de TIC, lo que indicaría la necesidad de seguir trabajando en el entendimiento, divulgación y afianzamiento de estos espacios (Borgobello, Sartori y Roselli, 2016). En las experiencias de este tipo en la UNR se advierte el predominio de sistemas bimodales o blended learning, en los que se combina lo presencial y lo virtual (Copertari, Sgreccia y Fantasía, 2014). En cuanto a las universidades privadas locales, no se cuenta con datos publicados, pero se conoce que disponen de entornos virtuales instalados, a disposición de los docentes y estudiantes de dichas instituciones y que su uso también es escaso.

La UNR es una institución con ingreso irrestricto y masivo en la mayor parte de las carreras de grado. Esta masividad requiere reforzar propuestas didáctico-pedagógicas más inclusivas y democráticas (Copertari, 2010). En este sentido, la modalidad de enseñanza y aprendizaje basada en educación a distancia democratizaría la educación superior y la haría más inclusiva, tanto en carreras de grado como de posgrado (Copertari et al., 2014).España y Foresi (2009) plantean que se ha generalizado el re- conocimiento de que el uso de TIC favorece el aprendizaje cooperativo y desarrolla nuevas habilidades de comunicación y creatividad, entre otras ventajas. Sin embargo, además de estar convencidos de la importancia de su implementación, para que las TIC se integren en la cotidianeidad de las prácticas docentes, tendrían que ser consideradas un aspecto a incluir en la formación profesional docente, para que su uso se lleve a cabo fundamentadamente y no con base en un conocimiento meramente intuitivo. En el contexto analizado, aún existe el preconcepto de que es posible apropiarse de conocimientos complejos sin mediación ni ayuda pedagógica (Sanjurjo, 2011). La naturaleza propia de la mediación a distancia requiere de materiales didácticos más objetivados que en esos medios reemplazan la "etérea verborragia del docente presencial” (Pozo, 2010, p.14).

Así, el proyecto en su conjunto se enmarca en la preocupación por entender qué relaciones sociocognitivas establecen docentes y estudiantes en contextos universitarios renovados por la incorporación del uso de TIC. En el enfoque de investigación adoptado, el aprendizaje en el contexto formal se da en interacción permanente con otros, directa o indirectamente, es decir, con otras personas o con materiales que han sido elaborados por otras personas. El análisis de la interacción sociocognitiva, así entendido, postula que cualquier práctica de enseñanza es social y cognitiva al mismo tiempo (Borgobello et al., 2016). Tal como lo expresa Roselli (2009), el socioconstructivismo cognitivo de raíz vigotskiana propone una aproximación cognitivamente interaccionista que supera la idea individual de aprendizaje vinculada con la psicología cognitiva clásica.

Otra categoría fundante de la investigación es la de buenas prácticas docentes en la enseñanza universitaria. Se trabajó con un criterio compartido con Zabalza (2012), quien plantea que el estudio de las buenas prácticas tendría que estar asociado a un doble propósito: por un lado, descriptivo y analítico y, por otro, transformador, por tanto, vinculado a la mejora de las prácticas docentes universitarias 
que son su objeto de estudio. La Figura 1 sintetiza las condiciones del análisis de las buenas prácticas, efectuado en la investigación en docencia universitaria. Tal como sintetiza Sanjurjo (2011, p.73):

entiendo por buenas prácticas aquellas que proponen y provocan procesos reflexivos, generan el aprendizaje de relaciones activas con el conocimiento, buscan la comprensión y apropiación significativa por parte de los estudiantes. Todo ello favorecido por un buen clima de aula, por la autoridad del docente ganada a través de su trato respetuoso y de sus conocimientos expertos.

\section{Figura 1. Condiciones para entender las buenas prácticas en docencia universitaria}

\begin{tabular}{|l|l|}
\hline Condiciones de las buenas prácticas \\
Como prácticas & no son principistas \\
Son visibles & por tanto, no implícitas \\
Son contextuales & es decir, no universalistas
\end{tabular}

Fuente: Zabalza (2012).

Las ideas sobre buenas prácticas educativas no fueron concebidas con relación a las TIC, pero de hecho pueden considerarse antecesoras en muchos sentidos. En la actualidad, son dos temas que suelen estar vinculados, dada la imbricación que ambos permiten, tanto a nivel conceptual como respecto de las prácticas docentes concretas del día a día.

\section{Diálogos entre conceptos teóricos y prácticas docentes}

Como podrá advertirse en los apartados subsiguientes, los temas planteados en los ejes que describen los autores representan preocupaciones que aparecen en la narrativa de los docentes, a partir tanto de los planteos iniciados en la guía de entrevistas como de la relación con el análisis que los propios docentes introducen en respuesta a las preguntas que se les formularon.

El guion de las entrevistas iniciales fue confeccionado con preguntas abiertas y cerradas. Habitualmente, las preguntas eran realizadas primero de modo abierto y luego se les pedía a los docentes que puntuaran u ordenaran las categorías que se les dieron. Las preguntas abordaron temáticas relativas al trabajo con los estudiantes, a la docencia universitaria, al contexto de trabajo y a las dificultades técnicas y de gestión de los entornos virtuales, entre otras. En este texto, por razones de extensión, solo se analiza parte de las entrevistas iniciales, específicamente las temáticas centradas en los estudiantes.

La construcción de algunas preguntas se basó en conceptos teóricos para indagar las nociones de buenas prácticas, trabajo colaborativo grupal (TGC) y estilos de aprendizaje. La idea era propiciar el diálogo de los docentes con teorías existentes, para posibilitar desde allí la problematización de sus propias prácticas. Por esta razón, en los apartados de análisis de las respuestas dadas por los docentes se incluyeron algunas descripciones teóricas densas. Así, se agruparon las respuestas en tres categorías de análisis:

- Ideas acerca de estudiantes ideales y habituales.

- $\quad$ Concepciones acerca del TCG.

- Temores y dificultades en relación con el uso de la plataforma.

En síntesis, aquí se analiza la narrativa de los docentes sobre las respuestas abiertas y se ilustra con tablas las respuestas cerradas.

Debido a la lógica dialéctica compleja (Achilli, 2005) a la que se adhiere y la diversidad de temas abordados en las entrevistas, en la construcción del texto no se separan antecedentes, método, resultados y discusión de una manera ortodoxa. En cada una de las tres grandes categorías de análisis describimos tanto las ideas y conceptos en las que se basó la construcción de cada pregunta, en diálogo con otros autores, como con los resultados de esta investigación, indagando otros escritos para abrir nuevos diálogos. Como consecuencia, la escritura reviste una forma fundamentalmente ensayística, aunque 
ISSN 0123-1294 | e-ISSN 2027-5358 | Educ.Educ. Vol. 21. No. 1 | Enero-abril de 2018 | pp. 27-48.

Universidad de La Sabana | Facultad de Educación

se torna informe de investigación y relato de experiencia en algunos momentos (Temporetti, 2005).

\section{Descripciones acerca de estudiantes ideales y habituales}

En el contexto analizado, y como uno de los resultados de esta investigación, se encontró que la concepción de estudiante ideal difiere radicalmente de la descripción que los docentes hacen de los estudiantes con que se encuentran habitualmente en las clases universitarias. Definir qué es un buen estudiante no es fácil, ya que depende de múltiples dimensiones. Falsafi y Coll (2011) se preguntan si es quien responde a las expectativas formales de un curso, quien aprueba con el menor esfuerzo posible, quien aprende de memoria o quien es capaz de ser crítico de los contenidos. Más allá de qué se entienda por buen alumno, los autores sostienen que las instituciones educativas deben prestar atención especial a la construcción y reconstrucción que los estudiantes hacen de la identidad de aprendiz.

Los estudiantes universitarios "ideales" son los que Casco (2009) describiría como afiliados institucionalmente: quienes desarrollaron la capacidad de "arreglárselas solos" en un medio que es poco estructurante. La autonomía, definida por la autora como una suprarregla de la vida universitaria, exige en el nivel intelectual un saber legítimo y validado, el uso de categorizaciones propias del mundo intelectual y capacidad de demostrar dominio del trabajo intelectual. Observa que los ingresantes a la universidad leen de modo literal, limitados al contenido, y escriben extrayendo y reproduciendo. Lejos del estudiante ideal esperado por la cultura académica, la población estudiantil "real" muestra una dependencia acrítica de los discursos. Sin embargo, destaca que es responsabilidad de la universidad, a través de sus esfuerzos pedagógicos, proveerles oportunidades para construir una relación diferente con los discursos, propiciando así procesos de desenvolvimiento auténtico de afiliación discursiva e intelectual. Ante esta situación, la institución tiene dos alternativas: deja al estudiante que ingresa librado a su propio esfuerzo o interviene con acciones específicas. Estas ideas aparecen en los decires de los docentes entrevistados, que muestran en algunas ocasiones su esfuerzo por afiliar a los estudiantes y, a veces, denotan signos de cansancio. Los propios estudiantes, según observa Pierella (2014), valoran el saber obtenido con el paso del tiempo, pero también critican la falta de adecuación de los docentes a las nuevas tecnologías y el cansancio de algunos profesores en el ejercicio de la docencia.

Para Huertas (2012), los malos estudiantes son quienes más necesitan estar motivados para el aprendizaje, pero estos no piden autonomía respecto del docente, sino cercanía socioafectiva, y necesitan un clima emocional, reconocimiento, evaluación permanente y retroalimentación. Sin embargo, a los buenos estudiantes también les importa ser guiados por el docente, pero quieren elegir y necesitan grados de libertad, ya que no les gusta hacer esfuerzos sin sentido. García y Blanco (2015) entrevistaron a estudiantes de formación profesional que mostraron que dificultades como baja autoestima, desinterés por contenidos curriculares y preferencia por actividades de ocio en las trayectorias académicas son reversibles. Estos estudiantes encontraron estímulo para superarse en sus familias y compañeros, pero escasamente en los docentes. No cuestionaron las limitaciones que imponían las instituciones, sino que se responsabilizaban a sí mismos de sus problemas debido a despreocupación, desorientación, entre otras razones.

Respecto al estudiante ideal en entornos virtuales, Alsina (2004) sostiene que, en este contexto, tanto el estudiante como el docente ideal son activos, participativos, se autoconducen y autoaprenden. El estudiante ideal tiene habilidades críticas para la navegación en internet, mientras que el profesor es facilitador del aprendizaje colaborativo y del trabajo grupal.

En consonancia con los resultados de este estudio, las instituciones de educación superior tienen 
como expectativa un estudiante ideal entregado a los estudios, aunque los cambios de los últimos tiempos han ampliado la distancia entre los requerimientos institucionales y las respuestas que los ingresantes tienen posibilidad de dar. Casco (2007) pone en evidencia la disonancia existente entre las reglas de la cultura universitaria y los valores, representaciones y saberes de los estudiantes. Estas diferencias detectan carencias y también contradicciones propias del sistema de enseñanza, que no favorece aprendizajes legítimos.

En un estudio de un curso blended learning se encontró que el grupo de estudiantes que se destacó por utilizar la plataforma más frecuentemente solo representaba un porcentaje cercano al 10\%. Estos estudiantes afirmaron que las explicaciones de los docentes eran muy claras, probablemente porque al utilizar más la plataforma se encontrarían más familiarizados que los otros compañeros con los términos específicos de la materia, retrabajados en explicaciones presenciales. Un segundo grupo representaba al $40 \%$ y se caracterizaba por tener el más bajo índice de actividad en el entorno virtual. Pero entre los estudiantes "ideales" y los menos activos, se encontraban casi la mitad del grupo, quienes hicieron un uso intermedio de la plataforma, a pesar de haber hecho evaluaciones positivas del entorno. Asimismo, prefirieron los cuestionarios, como la herramienta -dentro de las posibilidades brindadas por Moodle- que podría ser considerada la menos colaborativa. Las diferencias más importantes en los mensajes escritos por sujetos con bajo y alto rendimiento general estuvieron en la cantidad de mensajes escritos, ya que los estudiantes con más alto índice general de rendimiento académico (IGRA), si bien con variaciones individuales, sextuplicaron a los de más bajo IGRA. Las características de los mensajes escritos en foros mostraban diferencias significativas con respecto al contenido socioafectivo y organizativo y a la cantidad de respuestas obtenidas de otros compañeros en sus mensajes en foros. Asimismo, solo algunos estudiantes con rendimiento alto escri- bieron en foros optativos y usaron la primera persona en sus respuestas (Borgobello y Roselli, 2016).

En este estudio, al preguntarles a los docentes cómo eran sus estudiantes ideales en clases presenciales, se encontraron respuestas que los definían, según puede observarse en el Recuadro 1, como activos e interactivos con la figura del docente, y problematizadores, curiosos, responsables y capaces de esforzarse, es decir, afiliados institucionalmente a la universidad, en el sentido descrito por Casco (2009). Tal como podrá verse en el apartado siguiente, esta imagen del estudiante ideal dista de la descripción que los docentes hacen de los estudiantes que habitan cotidianamente las aulas universitarias.

Cabe señalar que solo uno de los docentes rescata la idea de la interacción entre los estudiantes como parte de las características de su ideal. El resto destaca solo la interacción basada en la relación docente-estudiante, como si se tratara de una relación dual y no colectiva.

\section{Recuadro 1. Caracterizaciones de los docentes acerca del estudiante ideal en clases tradicionales}

"[Es aquel] que se preocupa y [al] que le interesa lo que está haciendo, que muestra cierta responsabilidad hacia lo que está aprendiendo [...] es el estudiante que hace esfuerzos para integrar"; "Es el que lleva el material leído y el que logra posicionarse ante el material, el que lo problematiza [...] [en el que] ves que hay un interés [...]. Como docente, ojalá que todos puedan ser problematizadores."

"Es una persona proactiva y comprometida, no tanto con la materia y conmigo, sino a nivel social [...] [es alguien que] busca por su cuenta o trae problemáticas él mismo, tiene compromiso hacia lo que eligió y le genera curiosidad y motivación para buscar sus propias herramientas además de las que yo trato de darle."

"[Es el que] lee lo hay que leer para la clase y pregunta, pero porque está realmente interesado y no porque pregunta para figurar."

"[Es el que] no tiene miedo de preguntar [...] [con] ganas de aprender. Me preocupa más el chico del que no sé nada. Les digo a los chicos que no me interesa que me digan todo "excelente". Si yo pregunto algo es para que se equivoquen acá conmigo."

"El estudiante ideal no existe, es de cada uno para mí es el que lee aunque sea lo básico, pregunta, discute, te pone un poco contra la pared en cuanto a tus propios conocimientos como docente y te hace revisar temas, reflexionar, cuestionarte. El estudiante real es otro."

"[Yo] prefiero el caos, que los estudiantes charlen entre ellos, [...] que interactúen con el docente, [...] que intervengan todo el tiempo con lo que yo estoy diciendo y pueda tener un feedback."

Fuente: elaboración propia. 
ISSN 0123-1294 | e-ISSN 2027-5358 | Educ.Educ. Vol. 21. No. 1 | Enero-abril de 2018 | pp. 27-48.

Universidad de La Sabana | Facultad de Educación

Sintéticamente, el estudiante ideal en clases tradicionales es presentado como atento, responsable y problematizador. Adicionalmente a estas maneras clásicas de entender al estudiante ideal y real, ahora las TIC complejizan el panorama académico. Una pregunta que permitió el trabajo conjunto entre investigadoras y docentes entrevistados fue acerca de cómo diferencian el trabajo en las aulas tradicionales presenciales y lo que esperan del trabajo en el espacio digital. Al preguntarles a los docentes cómo consideraban a un estudiante ideal específicamente en entornos virtuales, la mayoría respondieron muy similar a como lo hicieron respecto de la idea de estudiante ideal en clases tradicionales, es decir, activo, interesado, responsable, curioso, etc. Cabe señalar que una de las docentes expresa la idea de colaborativo, que no había surgido con claridad en las percepciones anteriores.

Por otro lado, aquí el ideal contempla el acceso a una computadora conectada a internet en su domicilio. Es decir, si bien en la actualidad las instituciones a las que pertenecen los docentes entrevistados tienen computadoras con acceso a internet libre para los estudiantes, a priori, marcaría una diferencia importante el hecho de tener acceso en su domicilio para la interacción activa y espontánea en el aula virtual. Más allá de que los teléfonos móviles permiten acceder a entornos virtuales casi de modo continuo, aparentemente, la comodidad no sería la misma.

\section{Recuadro 2. Caracterizaciones de los docentes acerca del estudiante ideal en la plataforma virtual}

"[Es aquel] que participa [...], que trata [...], que colabora [...], el estudiante colaborativo, activo, cognoscente."

"[Es aquel] que por lo menos se involucra cuando vos proponés, el que se interesa, [...] responde, no abúlico. No sé si [sea el] que conozca tanto la tecnología, pero por lo menos [es el] que con las herramientas que tenga quiera participar."

"[Es el que] me gustaría que esté genuinamente interesado en la interacción. [...] Oue no se mida tanto en qué tipo de comentarios hace, sino que haga comentarios."

"[Es aquel] que tiene curiosidad y el compromiso. [...] obviamente tiene que tener acceso a una computadora porque no sé si todos los estudiantes tienen."
"[Ha de tener] las mismas características: que participe, que lea, supongo, que ingrese a la plataforma y que haga las actividades [...] creo que [así] se nota más quién participa."

"[Será aquel] que no tenga miedo a experimentar, que se involucre, que sea curioso, que sea inquieto. [...] El ideal virtual volvería a ver qué le dijo la docente, para ver si me sirve revisar los conceptos."

"Creo que es el que participa en la medida en que lo necesita y que puede aportar, no el pesado que está todo el tiempo tratando de lucirse sino que lo utiliza como una herramienta más para aprender."

Fuente: elaboración propia.

Luego se les preguntó cómo describirían a sus estudiantes habituales, aclarando que se pretendía que describieran las características comunes a la mayoría y no las excepciones que toda aula presenta. Tal como se ilustra en el Recuadro 3, solo algunos docentes muestran una visión positiva de sus estudiantes reales. La mayoría mencionan características estrictamente negativas y algunos rescatan aspectos favorables, en un esfuerzo para valorar positivamente algo en su descripción. Asimismo, dos de ellos destacan el esfuerzo como docente para que los estudiantes logren "encontrarle la vuelta", como esfuerzo pedagógico, en el sentido de Casco (2009) y de Huertas (2012). Las que siguen son algunas de las frases que más se destacaron.

\section{Recuadro 3. Caracterizaciones de los docentes acerca de los estudiantes habituales}

"Unas amebas, el 80\% de ellos son unas amebas [risas] [...] no les interesa [...] casi a fin de año ya [...] cuando me esforcé muchísimo para que lo logren, ahí empiezan entonces a encontrarle la vuelta."

"Mis estudiantes habituales leen y reproducen."

"No son problematizadores [...] Se quedan mucho con lo que dice el autor o con lo que opina el docente[...] como criterio de verdad [...], sin poder generar cosas nuevas o ser críticos."

"Va a las clases, le gusta que le hablen, que le digan lo que tiene que hacer, la mayoría de las veces no hace ni siquiera lo que tiene que hacer."

"[El estudiante] tiende a la 'ley del menor esfuerzo'."

"Cuesta mucho despertar la motivación en los chicos. Siempre se tiende a negociar."

"Son estudiantes que si les pido que me manden algo por mail, en general lo hacen, no necesariamente eso quiere decir que lo hagan en tiempo y en forma, pero lo hacen, no todos, pero sí la mayoría."

"Gente que interactuaba mucho conmigo en la clase y fuera de la clase."

"En promedio son estudiantes participativos, también yo tiendo a que sea bastante participativa la clase."

Fuente: elaboración propia. 


\section{Optar por describir: estudiantes ideales y habituales}

Con base en estudios sobre estilos de aprendizaje en estudiantes universitarios, se solicitó a los docentes que describan a sus estudiantes reales e ideales utilizando opciones dicotómicas, todas ellas positivas. Es decir, se trataba de diferentes aspectos descriptivos del aprendizaje. Se les preguntó cómo describirían a su estudiante ideal en clases presenciales / estudiante ideal en entornos virtuales / estudiante habitual con dos opciones dicotómicas:

- Predominantemente orientado a la experiencia concreta o hacia la conceptualización abstracta.

- $\quad$ Predominantemente orientado a la experimentación activa o a la observación reflexiva.

Estas categorías fueron tomadas del análisis de estilos de aprendizaje basada en estudios realizados a partir de cuestionarios, no de entrevistas (cfr. Ventura, 2013, 2016). Si bien las categorías surgen de otro tipo de estudio, al no estar orientadas a descripciones valorativas per se, ponen al docente en la situación de optar por lo que él entiende que describe de modo más adecuado a su estudiante, ya sea ideal o real, presencial o virtual. Además, metodológicamente, ello permitió comparar las respuestas obtenidas con categorías cerradas, complementando así la narrativa de los docentes. El modelo de Kolb establece que para aprender es necesario disponer de cuatro capacidades básicas: experiencia concreta (EC), observación reflexiva (OR), conceptualización abstracta (CA) y experimentación activa (EA), de cuya combinación surgen los cuatro estilos de aprendizaje propuestos por este modelo.

Construimos la Tabla 1 a partir de la combinación de respuestas a las dos preguntas en estudiantes ideales y habituales. Como puede observarse, la mitad de los docentes de este grupo describieron a los estudiantes habituales como asimiladores, es decir, según el modelo teórico, tendrían como características la conceptualización abstracta (CA) y la observación reflexiva (OR), las habilidades asociadas a la creación de modelos teóricos y un razonamiento inductivo. Por otro lado, la tendencia del grupo (con 6 respuestas en total) fue describir a los estudiantes ideales como convergentes en la conceptualización abstracta y la experimentación activa (EA), por tanto, serían personas que tenderían a la aplicación

Figura 2. Estilos de aprendizaje de Kolb*

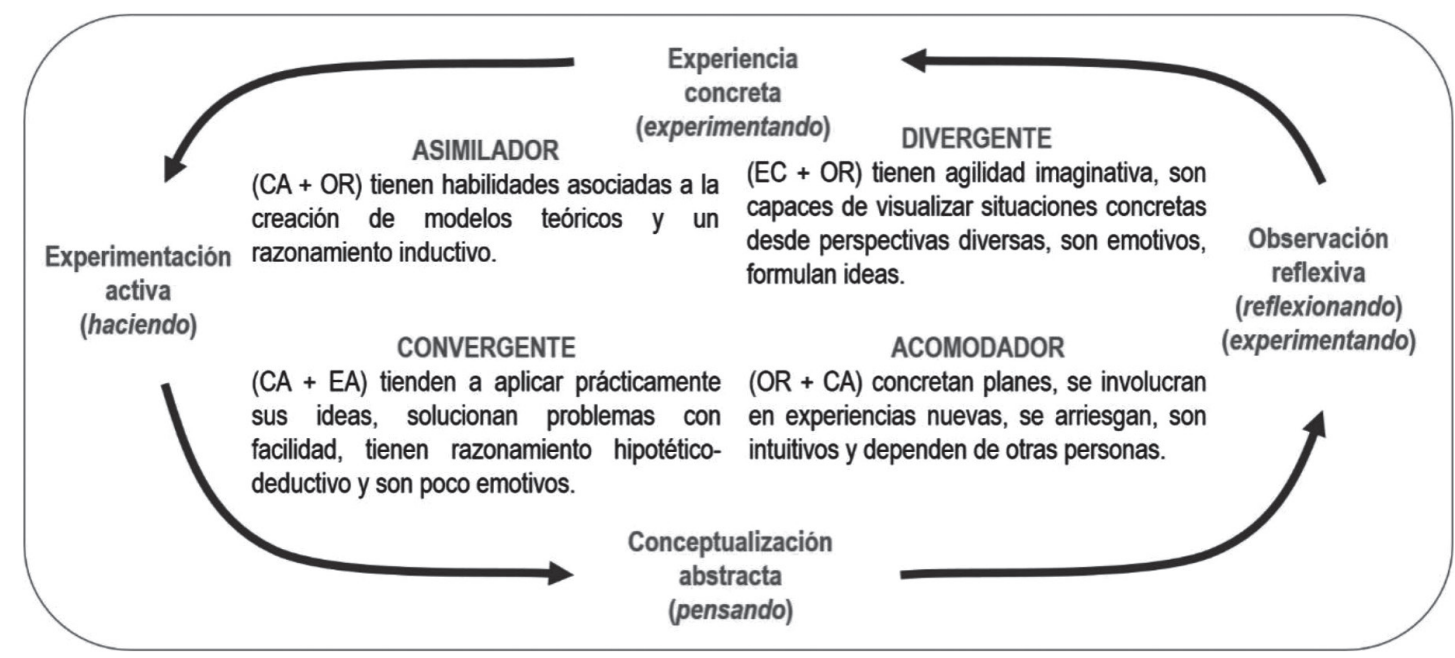

* $\quad \mathrm{EC}=$ experiencia concreta, $\mathrm{CA}=$ conceptualización abstracta, $\mathrm{EA}=$ experimentación activa, $\mathrm{OR}=$ observación reflexiva. Fuente: Romero, Salinas y Mortera (2010). 
ISSN 0123-1294 | e-ISSN 2027-5358 | Educ.Educ. Vol. 21. No. 1 | Enero-abril de 2018 | pp. 27-48.

Universidad de La Sabana | Facultad de Educación

práctica de sus ideas y a solucionar problemas con facilidad, con razonamiento hipotético-deductivo, y serían poco emotivos. Tres de los docentes coinciden en su descripción del estudiante ideal y habitual.

\section{Tabla 1. Estudiante ideal frente a los estudiantes} habituales en clases tradicionales

\begin{tabular}{|c|c|c|c|c|c|c|}
\hline & \multicolumn{5}{|c|}{ Estudiante ideal } \\
\hline & & 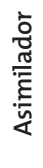 & 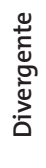 & 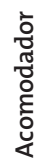 & 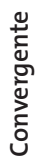 & 坖 \\
\hline \multirow{4}{*}{$\begin{array}{c}\text { Estudiantes } \\
\text { habituales }\end{array}$} & Asimiladores & - & 1 & - & 4 & 5 \\
\hline & Divergentes & - & - & 1 & - & 1 \\
\hline & Acomodadores & - & - & 1 & - & 1 \\
\hline & Convergentes & - & 1 & - & 2 & 3 \\
\hline Total & & 0 & 2 & 2 & 6 & 10 \\
\hline
\end{tabular}

Fuente: elaboración propia.

En relación con lo expresado, solo en tres casos los docentes describieron a sus estudiantes promedio con sus estudiantes ideales esperables en el uso de plataforma (Tabla 2).

Tabla 2. Estudiantes habituales frente al estudiante ideal en entorno virtual

\begin{tabular}{|c|c|c|c|c|c|c|}
\hline & \multicolumn{5}{|c|}{ Estudiante ideal } \\
\hline & & 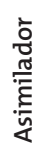 & 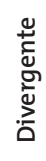 & 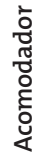 & 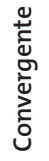 & 㞼 \\
\hline \multirow{4}{*}{$\begin{array}{c}\text { Estudiantes } \\
\text { habituales }\end{array}$} & Asimiladores & - & - & - & 5 & 5 \\
\hline & Divergentes & - & - & 1 & - & 1 \\
\hline & Acomodadores & - & - & - & 1 & 1 \\
\hline & Convergentes & - & - & - & 3 & 3 \\
\hline Total & & - & - & 1 & 9 & 10 \\
\hline
\end{tabular}

Fuente: elaboración propia.

En relación con los estudiantes ideales y el uso que hacen de la plataforma virtual, 9 docentes coincidieron en que deberían ser convergentes, es decir, con tendencia a la experiencia concreta y a la observación reflexiva, y solo 6 consideraron que sus estudiantes ideales en aulas presenciales debían ser convergentes (Tabla 3). Es decir, para algunos de los docentes, los estudiantes ideales en el uso de entornos virtuales y en las aulas tradicionales presentarían características distintas. Se observó una tendencia bastante homogénea respecto al estudiante ideal en entornos virtuales. Es posible que esta tendencia esté asociada a la escasa práctica en el uso de plataforma.

Tabla 3. Estudiantes ideales en clase tradicional y en entorno virtual

\begin{tabular}{|c|c|c|c|c|c|c|}
\hline & \multicolumn{5}{|c|}{ En entorno virtual } \\
\hline & & 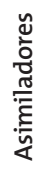 & 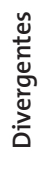 & 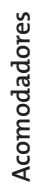 & 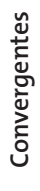 & 胥 \\
\hline \multirow{4}{*}{$\begin{array}{c}\text { EN CLASE } \\
\text { TRADICIONAL }\end{array}$} & Asimiladores & - & - & - & - & - \\
\hline & Divergentes & - & - & - & 2 & 2 \\
\hline & Acomodadores & - & - & 1 & 1 & 2 \\
\hline & Convergentes & - & - & - & 6 & 6 \\
\hline Total & & - & - & 1 & 9 & 10 \\
\hline
\end{tabular}

Fuente: elaboración propia.

Cabe destacar que, si bien no se pidió a los docentes que justificasen sus elecciones, algunos de ellos hicieron comentarios espontáneos que describen cabalmente las sensaciones ante las preguntas y lo que realmente piensan de sus estudiantes. A continuación, se ilustran algunos de los comentarios que complementan las tablas anteriores.

Refiriéndose al estudiante ideal en clases tradicionales, tres docentes justificaron su elección de "experiencia concreta" diciendo, por ejemplo: "no podría elegir entre alguno de los dos, pero en la clase presencial me inclinaría por la experiencia concreta"; "yo tengo la idea de estudiante ideal de primer año, recién empiezan", o "es el que tiene la capacidad de aplicar esos conocimientos". Otros docentes, también refiriéndose al ideal presencial, muestran cierta tendencia de sus estudiantes a lo reflexivo, lo cual se refleja en las siguientes respuestas: "porque 
la reflexión es importante, pero la experimentación activa es fundamental para llevar a lo anterior", "yo prefiero la experiencia activa. Eso no quiere decir que esa experiencia no sea reflexiva", "también como docente me gustaría ser experimentadora activa en una clase y eso no quita que sea reflexiva". Algo interesante de estos dos últimos comentarios es que no son respuestas automáticas, sino meditadas, acerca de la descripción de sus estudiantes.

En relación con el estudiante habitual en clases tradicionales, a un docente la pregunta le causó risa y respondió: "conceptualización abstracta, pero creo que ninguna de las dos". Otro docente, optando por "observación reflexiva", agrega "tengamos fe que aunque sea reflexionan"; otro dijo: "Digamos que observación reflexiva [...] digamos [risas]" y "reflexiva en lo que es su conveniencia". Estos dos últimos agregados muestran una valoración negativa de los estudiantes habituales, mientras que un docente, ante esa dicotomía, muestra una visión positiva: "tienen un poco de todo [...] yo creo que la mayoría va hacia lo reflexivo".

Un entrevistado, que optó por "experimentación activa" en la descripción de sus estudiantes habituales, agregó un comentario negativo: "mucha reflexión no había"; mientras que otro complementó la misma opción con una frase positiva: "los estudiantes de la noche realmente querían estar ahí, estaban posicionados de otra manera, preguntaban y cuestionaban desde su recorrido". Si bien no se exploró en este estudio, investigaciones posteriores podrían indagar diferencias en las concepciones que los docentes tienen de sus estudiantes comparando años que cursan y bloques horarios.

\section{Concepciones acerca del trabajo colaborativo grupal}

Los usos pedagógicos que los participantes de un curso hacen de las TIC dependen tanto de las características de los recursos tecnológicos como de las actividades planificadas por los docentes, esto es, del diseño tecnopedagógico, atravesado por la tecnología utilizada y por la didáctica, de modo indisociable (Coll, 2004; Saz, Engel y Coll, 2016).

Como se mencionó anteriormente, reunimos una serie de preguntas de las entrevistas en un eje dedicado a las concepciones de los docentes sobre el TCG dado que la colaboración como concepto pedagógico atraviesa el diseño tecnopedagógico en el sentido de Coll (2004) y fue una de las la teorías centrales en la construcción de Moodle como entorno virtual (Llorente, 2007). Por esta razón, se construyeron preguntas específicas acerca de las ideas que los docentes tienen sobre TCG, además de sus hábitos pedagógicos en clases tradicionales, entre otras.

La idea de colaboración alude a situaciones en las que los sujetos trabajan con roles intercambiables, de modo horizontal y en conjunto, pudiendo aparecer alguna división espontánea del trabajo, que es horizontal, a diferencia de la cooperación, en la que la división del trabajo suele ser más "vertical" y fija. Por tanto, la colaboración es una estructura social en la que dos o más personas interactúan, que en circunstancias específicas tiene efectos positivos (Peralta y Borgobello, 2007).

Dillenbourg (1999) plantea que dicha interactividad la define el grado en que las interacciones influyen en los procesos cognitivos de los demás participantes y no la frecuencia de las interacciones. Nada garantiza que una auténtica interacción ocurra, pero existen maneras de aumentar las probabilidades de que se produzca. La sincronía de la tarea elegida, un adecuado diseño de la situación, el establecimiento de un andamiaje por parte de los docentes, reglas claras de interacción, una supervisión y regularidad en las interacciones, todo ello en conjunto puede promover la colaboración (Peralta y Borgobello, 2007). La plataforma Moodle, utilizada en esta experiencia, fue concebida conceptualmente, como se dijo, en relación con el aprendizaje colaborativo (Llorente, 2007). 
El guion de las entrevistas incluyó preguntas específicas sobre trabajo grupal, conflicto, aprendizaje colaborativo (véase Anexo 1). Se les pidió a los docentes que puntúen entre "Nunca" (1) y "Casi todas las clases" (5) la cantidad de trabajos grupales colaborativos en sus clases presenciales. Todos puntuaron entre 3 y 5 , es decir, en este grupo de docentes las propuestas pedagógicas habituales contemplaban TCG en gran medida. A su vez, los docentes dijeron cuán de acuerdo estaban con seis afirmaciones acerca del TCG. Las respuestas oscilaban entre "Muy en desacuerdo" (o) y "Muy de acuerdo" (4). La mitad de estas afirmaciones mostraban aspectos positivos del TCG y la otra mitad, aspectos negativos. A continuación se presentan las seis afirmaciones del guion de las entrevistas:

- (+) El TCG beneficia a la mayoría de los estudiantes.

- (-) El TCG desordena demasiado la clase y molesta al que realmente quiere estudiar.

- (+) El TCG estimula la creatividad, por el intercambio de ideas que produce.

- (-) El TCG genera estudiantes "parásitos" que se benefician del trabajo de otros.

- (-) El TCG implica más pérdida de tiempo que beneficios.

- (+) El TCG permite debatir distintos puntos de vista y ampliar el propio.

Un docente que estaba completamente en desacuerdo con las tres afirmaciones negativas acerca del TCG, sumaba o en este aspecto en la Tabla 4 (los docentes 5 y 9); un docente que opinaba que estaba "Muy de acuerdo" con las tres afirmaciones positivas sobre el tema sumaba 12 (docentes 4, 5, 8 y 9). Por tanto, la docente 5 puede considerarse la más optimista respecto del TCG, ya que suma 12 en la diferencia entre ambas columnas de la tabla.
Tabla 4. "Nivel de optimismo" respecto al trabajo colaborativo en grupos (TCG)

\begin{tabular}{|c|c|c|c|}
\hline & $\begin{array}{c}\text { Opiniones } \\
\text { sobre tópicos } \\
\text { negativos }\end{array}$ & $\begin{array}{c}\text { Opiniones } \\
\text { sobre tópicos } \\
\text { positivos }\end{array}$ & $\begin{array}{c}\text { Diferencias entre } \\
\text { negativos y } \\
\text { positivos } \\
\text { “Nivel de } \\
\text { optimismo" }\end{array}$ \\
\hline Docente 1 & 2 & 9 & 7 \\
\hline Docente 2 & 4 & 11 & 7 \\
\hline Docente 3 & 3 & 11 & 8 \\
\hline Docente 4 & 3 & 12 & 9 \\
\hline Docente 5 & 0 & 12 & 12 \\
\hline Docente 6 & 2 & 12 & 10 \\
\hline Docente 7 & 2 & 12 & 8 \\
\hline Docente 8 & 2 & 8 & 5 \\
\hline Docente 9 & 0 & 10 & 8,5 \\
\hline Docente 10 & 5 & 10,8 & 9 \\
\hline Promedio & 2,3 & & 10 \\
\hline
\end{tabular}

Fuente: elaboración propia.

En términos generales, este grupo de docentes fue optimista respecto de los beneficios que los estudiantes tienen al trabajar colaborativamente entre ellos. Se podría decir, a pesar de que tienen una visión predominantemente negativa de sus estudiantes habituales, que consideran que hacen una construcción planificada de sus clases en función de objetivos pedagógicos que fomentan el diálogo.

\section{Expectativas, temores y dificultades: entre la tecnología y la motivación}

Este eje de análisis fue construido dialécticamente a partir de las preguntas de la entrevista y de las respuestas espontáneas de los docentes. Por tanto, al eje de análisis de las dificultades y temores se agrega la motivación de los estudiantes como categoría emergente. En otras palabras, la motivación no formó parte de la guía construida para las entrevistas en ninguna de las preguntas cerradas.

Se les pidió que ordenasen de 1 a 5 las dificultades frecuentes en el uso de plataformas virtuales, siendo 1 la que intuían sería la más problemática y 5 la menos problemática al momento de su futura 
implementación. A partir de los datos obtenidos, se hizo un promedio de las respuestas ordinales -método que reconocemos como no ortodoxocon la intención de ilustrar opiniones comunes o cercanas en el grupo. Como puede apreciarse en la Tabla 5, el manejo técnico de la plataforma es la mayor dificultad percibida, seguida de la elección de la herramienta más adecuada, de acuerdo con el tema trabajado, dificultades que podrían apreciarse como habilidades vinculadas a la mediación tecnológica o tecnopedagógica, en el sentido señalado por Saz et al. (2016).

El seguimiento de las actividades parece ser la categoría menos preocupante en este grupo de docentes. La opción elegida como menos conflictiva estuvo vinculada con lo específicamente pedagógico, es decir, con lo que aparentemente no está relacionado en forma directa con la tecnología.

Posteriormente se indagó acerca de otras posibles dificultades no mencionadas. Seis de los docentes no mencionaron dificultades adicionales, mientras que quienes lo hicieron se refirieron a dificultades en la motivación y responsabilidad de los estudiantes, tema que apareció como una preocupación recurrente en las entrevistas, tanto ante esta pregunta como en otras, y que se manifestaron, por ejemplo, en las siguientes percepciones, según las cuales la dificultad está "en que los chicos se 'copen", "en motivarlos [...] [por lo que] me va a ser difícil que ellos respondan o participen", en "motivar a los estudiantes para usar la plataforma", en "que los estudiantes no quieran ingresar a la plataforma", en "el atraso en las actividades, que se cuelguen", y un docente expresó lo siguiente: "no me sirve que trabajen obligados, pero es necesario poner alguna obligatoriedad en el uso para que surja la motivación".

Cabe destacar que, en el contexto local de la UNR, pueden encontrarse docentes reconocidos y con amplia trayectoria que consideren que los estudiantes de las nuevas generaciones están menos motivados por el estudio y tienen menos autoexigencia que generaciones de décadas anteriores (Pierella, 2015).

Si bien definir motivación resulta una tarea compleja, existe acuerdo en relacionarla con la elección de una acción, la persistencia en esa acción y el esfuerzo para que se lleve adelante. Específicamente en contextos académicos, la motivación para los estudiantes implicaría elegir una tarea, persistir en su realización y esforzarse para resolverla (Chiecher, Donolo y Rinaudo, 2004).

Existen diferentes perspectivas teóricas y abordajes de la motivación. Desde una perspectiva cognitiva que refiere a las características intrínsecas de los sujetos, el docente es acompañante de un proceso que es individual. Por ejemplo, Garrote, Garrote y Jiménez (2016) plantean que los estudiantes, al sentirse competentes, autónomos, en control de sus acciones e involucrados en la tarea, consideran la motivación como satisfactoria y de interés. El hecho de que se encuentren altamente involucrados favorece la consecución de los objetivos académicos. En todo ello, sostienen los autores, se debe acompañarlos para el uso de técnicas de estudio adecuadas y con métodos de enseñanza y evaluación acordes.

Tabla 5. Dificultades identificadas a priori en el trabajo con la plataforma

\begin{tabular}{|l|c|c|c|c|c|c|c|c|c|c|c|}
\hline & D1 & D2 & D3 & D4 & D5 & D6 & D7 & D8 & D9 & D10 & Pro-medio \\
\hline En el manejo técnico de la plataforma (software) & 5 & 2 & 4 & 2 & 1 & 5 & 1 & 1 & 1 & 3 & 2,5 \\
\hline En la elección de la herramienta más adecuada al tema & 2 & 1 & 3 & 5 & 2 & 1 & 4 & 2 & 4 & 2 & 2,6 \\
\hline En la evaluación de las actividades que realicen los estudiantes & 4 & 4 & 1 & 1 & 4 & 3 & 3 & 4 & 3 & 4 & 3,1 \\
\hline En el seguimiento de las actividades de los estudiantes & 1 & 5 & 5 & 4 & 3 & 2 & 2 & 5 & 5 & 1 & 3,3 \\
\hline En la elaboración de las consignas de trabajo & 3 & 3 & 2 & 3 & 5 & 4 & 5 & 3 & 2 & 5 & 3,5 \\
\hline
\end{tabular}

Fuente: elaboración propia. 
Desde otra perspectiva que incluye como dimensión de análisis lo institucional, el docente juega un rol decisivo y, por ende, más activo. En un análisis sobre hábitos de estudio y motivación, Acevedo, Torres y Tirado (2015) muestran que los estudiantes tienen escasa motivación, métodos de estudio poco adecuados, escasa planificación de las actividades académicas y una inadecuada distribución del tiempo. Por ello proponen que los docentes desarrollen talleres $u$ otros sistemas para que los estudiantes puedan mejorar sus técnicas de estudio, ya que no basta con que las conozcan y es necesario que puedan relacionarlas con el tipo particular de material, los objetivos del aprendizaje a lograr y el nivel de conocimiento propio del tema que se trate.

Así también, Canales y Marquès (2008) sostienen que es necesario que los estudiantes encuentren sentido en las actividades para que estén motivados a utilizar los recursos para aprender. En este sentido, destacan que los estudiantes asocian el uso de TIC más al ocio que a la formación académica. Sin embargo, también remarcan que para el uso de TIC el protagonismo, el impulso y la motivación inicial deberían surgir de los propios docentes.

Copertari et al. (2014) señalan la resignificación del rol docente en contextos blended learning, lo cual propicia estilos de aprendizaje flexibles y favorece la orientación a través de múltiples direcciones para la comunicación. Así se da la posibilidad de aumentar la motivación de los estudiantes y la creatividad.

Se desconoce si la percepción del contexto es la que predispone a una mayor motivación o es el hecho de estar más motivado lo que favorece una mejor percepción del contexto. En un estudio conducido por Chiecher et al. (2004), los estudiantes con mejores percepciones del contexto informaban patrones motivacionales más adaptativos. De todos modos, los autores destacan la importancia de ofrecer a los estudiantes entornos con recursos variados, amigables, ricos en estímulos y relajados, para intentar incidir en su motivación.
Guzmán (2011) afirma que explicitar los beneficios de aprender lo que se está enseñando favorecería la motivación de los estudiantes. Los profesores perciben que buenas prácticas como la mencionada tienen incidencias en una mayor implicación de los estudiantes en las actividades de aprendizaje (Valverde, Fernández y Revuelta, 2013).

Para aprender, "las TIC generan motivación debido a que permiten trabajar en entornos basados en lenguajes iconográfico-visuales y porque favorecen el trabajo autónomo de los propios estudiantes" (Garrido, Contreras y Miranda, 2013, p.66). Esta idea se cuenta entre las cinco creencias centrales que poseen los estudiantes de carreras de pedagogía acerca de las tecnologías digitales en la formación.

Las cuestiones que inciden en la motivación, compromiso y esfuerzo de los estudiantes tienen múltiples causales, siendo la comunicación una de las aristas a tener en cuenta. Algunos estudiantes disconformes con los estilos tradicionales de enseñanza imperantes en las culturas docentes universitarias manifiestan sentirse intimidados a expresarse al respecto, diciendo, por ejemplo, "los profesores dicen querer debates pero cuando no opinas lo que quieren ellos no les gusta" (Álvarez, Rodríguez y Maroto, 2013, p.139).

Los estudiantes universitarios destacan la motivación o "enganche" que producen docentes destacados de la UNR, a quienes atribuyen autoridad relacionada con un proceso que Pierella (2014) destaca como característica de seducción. En este sentido, la autora marca que el "saber enseñar" es visto por los estudiantes como algo individual, un "don" o talento que poseerían solo algunos profesores. Uno de los docentes entrevistados por Pierella (2015, p.38) explica su interés por motivar la curiosidad en los estudiantes recurriendo al recuerdo de uno de sus docentes de antaño: "la manera en que nos planteaba las preguntas y cómo nos motivaba a la curiosidad fue para mí determinante. Fue reencontrar ese placer que de alguna manera tenía de niño, reencontrarme con esa manera de acceder a la pregunta y a la búsqueda de la respuesta". 
Las exceptivas ante los cambios, los temores y las dificultades frente a la incorporación de TIC, así como la relación entre motivación, uso de plataforma y enseñanza universitaria revisten, como puede advertirse, múltiples enfoques y perspectivas de análisis.

En sintesis, las concepciones acerca de los estudiantes, del trabajo colaborativo, del uso de TIC, junto con las dificultades de motivación en los estudiantes y docentes, como el rol institucional de las universidades ante estos cambios y problemáticas, implican posicionamientos político-académicos que tienen efectos en los diseños tecnopedagógicos.

\section{Discusión y conclusiones}

Cuando me refiero a posiciones crítico-salvajes acerca de la enseñanza estoy pensando en teorías y prácticas que, partiendo de una crítica a los enfoques tradicionales desembocan en la negación de toda intervención o ayuda pedagógica desde otras perspectivas. Toda intervención es considerada en si misma tradicional y nociva. El antimetodismo, antididactismo, antipedagogismo son presentados como únicas salidas "post-modernas" a los problemas de aprendizaje. Además de agregar oscuridad a la problemática, esta posición evidencia por un lado, un total desconocimiento de las profundas modificaciones epistemológicas en el campo de la Pedagogía y de la Didáctica. Y por otro, recae en posturas teóricas y prácticas que también desconocen los complejos procesos de mediación en la enseñanza y en el aprendizaje. Por esa misma razón promueven el regreso a prácticas tradicionales (Sanjurjo, 2011, p.77).

Tal como expresa Sanjurjo (2011), las prácticas tradicionales pueden sostenerse aún en posiciones consideradas como fuertemente críticas. Por ello, en este trabajo se procuró construir el análisis de los datos partiendo de la reflexión conjunta con los docentes asociada a las posibles buenas prácticas en enseñanza y aprendizaje de nivel universitario, específicamente con relación al inicio en el uso de la plataforma Moodle.
Tal como plantean Copertari et al. (2011), la virtualización de la universidad constituye una necesidad y se trata de un proceso técnico, pero esencialmente es un proceso político, social y cultural que implica acciones estratégicas y pone especial énfasis en la formación docente, por los desafíos pedagógicos que implican estas actividades. Cabe aclarar que en el guion de las entrevistas a los docentes no se han incorporado en forma directa ejes relativos a dimensiones políticas, institucionales y de gestión académica, por lo que en futuros trabajos sería interesante incluir estas dimensiones, para poder trabajarlas en conjunto con diferentes actores institucionales involucrados en el proceso.

En este sentido, España y Foresi (2009) cuestionan el papel de la tecnología en la enseñanza en el marco del sueño tecnocrático y mencionan que lo necesario es transformar las prácticas docentes, ya que la tecnología no es la que transforma. Por tanto, el uso de tecnologías de la información y la comunicación (TIC) no implica por sí mismo cambio y de hecho es posible reproducir prácticas tradicionales de enseñanza en entornos virtuales.

En el contexto del proyecto del cual se desprende el presente escrito, toma fuerza e interés la pregunta sobre las buenas prácticas en entornos virtuales. En el reconocimiento de que aún hoy su implementación es incipiente y las experiencias son aisladas en la UNR, se devela que todavía queda por trabajar en el entendimiento y afianzamiento de estos espacios (Borgobello et al., 2016). Desde esta perspectiva, hemos indagado cuáles son los temores y dificultades que tienen los docentes en la implementación de TIC, las concepciones y frecuencia del TCG en el aula e ideas respecto a los estudiantes ideales y habituales.

En esta línea de pensamiento, y a partir del trabajo con docentes considerados como reales coinvestigadores, aquí se analizaron diferentes ejes que, desde luego, no agotan la complejidad del tema, pero invitan a pensar e interrogar los desafíos que enfrenta actualmente la docencia universitaria y 
particularmente los atinentes a la implementación de entornos virtuales. Así, retomando a Zabalza (2012), el estudio de las buenas prácticas docentes tiene un propósito no solo descriptivo sino también $y$, ante todo, transformador.

En relación con lo planteado por los docentes entrevistados, se observó que una de las principales dificultades percibidas antes de la implementación de la plataforma virtual fue el manejo técnico de la misma, seguido de la elección de la herramienta más adecuada de acuerdo con el tema trabajado. Las opciones elegidas como menos dificultosas se vincularon a lo específicamente pedagógico, es decir, a lo que aparentemente no se relaciona con la tecnología. Así, es posible pensar las apreciaciones de los docentes acerca de lo que representa lo menos conflictivo como la zona más confortable dada la experiencia propia.

El grupo de docentes entrevistado fue optimista respecto de los beneficios que tiene para los estudiantes trabajar colaborativamente (el TCG). En este sentido, se podría decir que, si bien la percepción sobre los estudiantes es mayormente negativa, construyen sus clases con objetivos pedagógicos que fomentan el diálogo y la colaboración. Por otro lado, al indagar por las concepciones del estudiante ideal y real se encontraron importantes diferencias. Los docentes describieron al estudiante ideal empleando calificativos como: activo, problematizador, responsable. Muy por el contrario, los estudiantes habituales fueron considerados como de escasa participación, acríticos y tendientes a la "ley del menor esfuerzo".

A su vez, cuando los docentes debieron optar para describir y caracterizar al estudiante ideal y al habitual, tanto en clases tradicionales como en plataformas virtuales, se encontraron nuevamente apreciaciones negativas asociadas a las respuestas cerradas respecto al estudiante con que frecuentemente se encuentran en sus aulas. Es decir, luego de elegir una de las opciones, que no presentaban valores positivos o negativos, sino diferentes aspectos de los estilos de aprendizaje, los docentes expresaron de manera espontánea y de modo recurrente apreciaciones negativas.

En este mismo sentido, surgió en diversas ocasiones la problemática de la motivación de sus estudiantes para con las tareas académicas, es decir, el desafío con que se encuentran para que a sus estudiantes les guste la materia y trabajen. Así, resulta oportuno señalar que los estudiantes reales distan de ser descritos como afiliados institucionalmente a la universidad, en el sentido que lo entiende Casco (2009) y de responder cabalmente al modelo de Kolb descripto por Romero et al. (2010), algo que se vislumbra en los comentarios asociados a las opciones dicotómicas.

A partir de los puntos mencionados, y en una lógica inductiva aplicada al proceso de construcción de conocimiento, se llegó a reconsideraciones metodológicas. Las opciones dicotómicas presentadas a los docentes para sus descripciones debieran ser revisadas para la realización de futuras entrevistas en lo que respecta a los estudiantes habituales, ya que generaron respuestas asociadas a la elección dicotómica en disconformidad con las opciones presentadas. Quizá, al menos, debiera solicitarse algún tipo de justificación para la elección de cada categoría con el fin de transmitir las apreciaciones sin la restricción propia del tipo de pregunta.

El último eje de análisis del presente artículo fue construido dialécticamente entre las preguntas de la guía de entrevistas y las respuestas espontáneas, por lo que la categoría "motivación de los estudiantes" adquirió una relevancia que no había sido considerada previamente en el diseño del guion de la entrevista. En futuras investigaciones sería pertinente incorporar preguntas sobre el tema, debido a que esta preocupación fue recurrente en las distintas entrevistas y ante diferentes preguntas. 


\section{Referencias}

Acevedo, D., Torres, J. D. y Tirado, D. F. (2015). Análisis de los hábitos de estudio y motivación para el aprendizaje a distancia en estudiantes de Ingeniería de Sistemas de la Universidad de Cartagena (Colombia). Formación Universitaria, 8(5), 59-66.

Achilli, E. L. (2005). Investigar en la antropología social. Los desafios de transmitir un oficio. Rosario: Laborde.

Alsina, J. (2004). Utopías digitales y los entornos virtuales de aprendizaje. Reencuentro, 41(1), 1-12.

Álvarez, E., Rodríguez, A. y Maroto, J. L. S. F. (2013). Metodologías y recursos didácticos en los títulos de grado. ¿Qué hace el profesorado y qué quiere el alumnado? Tendencias pedagógicas, 22, 127-148.

Borgobello, A. y Roselli, N. D. (2016). Rendimiento académico e interacción sociocognitiva de estudiantes en un entorno virtual. Educação e Pesquisa, 42(2), 359-374.

Borgobello, A., Sartori, M. y Roselli, N. (2016). ¿Cómo interactuamos aquí y allá? Análisis de expresiones verbales en una clase presencial y otra virtual a partir de dos sistemas de codificación diferentes. Revista de La Educación Superior, 45(179), 95-110.

Bustos, A. y Coll, C. (2010). Los entornos virtuales como espacios de enseñanza y aprendizaje. Revista Mexicana de Investigación Educativa, 15(44), 163-184

Canales, R. y Marquès, P. (2008). Factores de buenas prácticas educativas con apoyo de las TIC. Análisis de su presencia en tres centros educativos. Educar, 39, 115-133.

Casco, M. (2007). Prácticas comunicativas del ingresante y afiliación intelectual. Ponencia presentada en el V Encuentro Nacional y II Latinoamericano “La Universidad como objeto de investigación”, Tandil, Argentina.

Casco, M. (2009). Afiliación intelectual y prácticas comunicativas de los ingresantes a la universidad. Co-herencia, 6(11), 233-260.

Coll, C. (2004). Psicología de la educación y prácticas educativas mediadas por las tecnologías de la información y la comunicación. Una mirada constructivista. Sinéctica, 25(1), 1-24.

Coll, C. y Sánchez, E. (2008). Presentación. El análisis de la interacción estudiante-profesor: líneas de investigación. Revista de Educación, 346, 15-32.

Copertari, S. (2010). La práctica docente universitaria en Educación a Distancia. Procesos metacognitivos y buena enseñanza. Rosario: Laborde.

Copertari, S., Sgreccia, N. y Segura, M. L. (2011). Políticas universitarias, gestión y formación docente en educación a distancia. Hacia una pedagogía de la virtualización. Revista de Educación a Distancia, 27, 1-16.

Copertari, S., Sgreccia, N. y Fantasía, Y. (2014). Educación a distancia: concepciones docentes y democratización de la enseñanza en los postgrados de la UNR. Sophia, 10(2), 23-34. 
ISSN 0123-1294 | e-ISSN 2027-5358 | Educ.Educ. Vol. 21. No. 1 | Enero-abril de 2018 | pp. 27-48.

Universidad de La Sabana | Facultad de Educación

Dillenbourg, P. (1999). What do you mean by collaborative learning? En P. Dillenbourg (ed.), Collaborative-learning: Cognitive and computational approaches (pp.1-19). Oxford: Elsevier.

Chiecher, A., Donolo, D. y Rinaudo, M. C. (2004). Estudiantes en entornos tradicionales y a distancia. Perfiles motivacionales y percepciones del contexto. Revista de Educación a Distancia, 3(10), 1-14.

España, A. E.y Foresi, M. F. (2009). Las prácticas y el desarrollo profesional ante las tecnologías de la información y de la comunicación. En L. Sanjurjo (coord.), Los dispositivos para la formación en las prácticas profesionales (pp.183-222). Rosario: Homo Sapiens.

Falsafi, L. y Coll, C. (2011). La construcción de la identidad de aprendiz: coordenadas espacio-temporales. En J. I. Pozo y C. Monereo (coords.), La identidad en Psicología de la Educación: necesidad, utilidad y límites (pp.7798). Barcelona: Narcea.

García, S. y Blanco, N. (2015). Los ciclos formativos de grado medio: una opción para salir del laberinto personal y académico. Tendencias Pedagógicas, 25(1), 301-320.

Garrido, J., Contreras, D.y Miranda, C. (2013). Análisis de la disposición pedagógica de los futuros profesores para usar las TIC. Estudios Pedagógicos, 34, 59-74.

Garrote, D., Garrote, C.y Jiménez, S. (2016). Factores influyentes en motivación y estrategias de aprendizaje en los estudiantes de grado. Revista Iberoamericana sobre Calidad, Eficacia y Cambio en Educación, 14(2), 31-44.

Guzmán, J. C. (2011). La calidad de la enseñanza en educación superior ¿Qué es una buena enseñanza en este nivel educativo? Perfiles Educativos, 33(1), 129-141.

Huertas, J. A. (2012). Los efectos de los afectos en la motivación y la autorregulación. Ciencias Psicológicas, 6(1), 45-55.

Llorente, M. C. (2007). Moodle como entorno virtual al alcance de todos. Comunicar, 15(28), 197-202.

Peralta, N. y Borgobello, A. (2007). Teoría del conflicto sociocognitivo y aprendizaje colaborativo en el ámbito universitario. Trabajo presentado en las XIV Jornadas de Investigación, Buenos Aires, Argentina.

Pierella, M. (2015). Universidad, conocimiento y transmisión. Un estudio centrado en biografías de profesores de carreras humanísticas y científicas. Revista D'innovació i Recerca en Educació, 8(1), 31-49.

Pierella, M. P. (2014). La autoridad de los profesores desde la perspectiva estudiantil. Revista Brasileira de Educação, 19(59), 893-912.

Pozo, M. I. (2010). Prólogo. En S. Copertari, La práctica docente universitaria en Educación a Distancia. Procesos metacognitivos y buena enseñanza (pp.13-14). Rosario: Laborde.

Romero, L. N., Salinas, V.y Mortera, F. J. (2010). Estilos de aprendizaje basados en el modelo de Kolb en la educación virtual. Apertura, 2(1), 1-21. 
Roselli, N. (2009). Intervenciones en contextos educativos microsociales para el desarrollo de la colaboración sociocognitiva. Revista Electrónica de Psicología Social Poiésis, 18(1), 1-5.

Sanjurjo, L. O. (2011). La clase: un espacio estructurante de la enseñanza. Revista de Educación, 3(2), 71-84.

Saz, A., Engel, A. y Coll, C. (2016). Introducing a personal learning environment in higher education. An analysis of connectivity. Digital Education Review, 29(1), 1-14.

Temporetti, F. (2005). Escritura y producción de conocimiento en psicología. En O.Menin y F.Temporetti (comps.), Reflexiones acerca de la escritura cientifica (pp.53-67). Rosario: Homo Sapiens.

Valverde, J., Fernández, M. R. y Revuelta, F. I. (2013). El bienestar subjetivo ante las buenas prácticas educativas con TIC: su influencia en profesorado innovador. Educación XXI, 16(1), 255-280.

Ventura, A. C. (2013). Estilos de aprendizaje y comunidades disciplinares desde un enfoque situado de la cognición. Revista Internacional de Psicología, 12(2), 1-29.

Ventura, A. C. (2016). ¿Enseño como aprendí?: el rol del estilo de aprendizaje en la enseñanza del profesorado universitario. Aula Abierta, 44(1), 91-98.

Zabalza, M. A. (2012). El estudio de las "buenas prácticas" docentes en la enseñanza universitaria. Revista de Docencia Universitaria, 10(1), 17-42.

Zúñiga, M. S. y Martínez, R. (2015). Los entornos virtuales: una necesidad en el mejoramiento de la dinámica del proceso de enseñanza-aprendizaje del Curso Presencial Intensivo en el ITB. Santiago, 138, 120-144. 


\section{Anexo 1.}

\section{Listado de preguntas analizadas en este artículo y aclaraciones relativas al instrumento utilizado}

1. ¿Cómo describirías a tu estudiante ideal en clases presenciales?

2. ¿Cómo te imaginás que sería un estudiante ideal en entornos virtuales?

3. ¿Cómo son tus estudiantes habituales?

4y 5. Tu estudiante ideal en clases presenciales está: a) ¿Orientado predominantemente hacia la experiencia concreta o hacia la conceptualización abstracta? y b) ¿Orientado predominantemente hacia la experimentación activa o hacia la observación reflexiva?

6 y 7. Un estudiante ideal en entornos virtuales está: a) ¿Orientado predominantemente hacia la experiencia concreta o hacia la conceptualización abstracta? y b) ¿Orientado predominantemente hacia la experimentación activa o hacia la observación reflexiva?

8 y 9. Tu estudiante habitual está: a) ¿Orientado predominantemente hacia la experiencia concreta o hacia la conceptualización abstracta? y b) ¿Orientado predominantemente hacia la experimentación activa o hacia la observación reflexiva?

10. ¿Es habitual que pidas a tus estudiantes trabajos grupales colaborativos (TCG)? (Los docentes optaban por el número que mejor representara su idea, siendo 1 "Nunca" y 5 "Casi todas las clases").

(En las preguntas 11 a 16 los docentes optaban por el número que mejor representara su idea, siendo 1 "Nada de acuerdo"y 5 "Muy de acuerdo".)

11. El TCG beneficia a la mayoría de los estudiantes.

12. El TCG desordena demasiado la clase y molesta al que realmente quiere estudiar.

13. El TCG estimula la creatividad por el intercambio de ideas que produce.

14. El TCG genera estudiantes "parásitos" que se benefician del trabajo de otros.

15. El TCG implica más pérdida de tiempo que beneficios.

16. El TCG permite debatir acerca de distintos puntos de vista y amplía el propio.

17. ¿En qué crees que encontrarás más dificultades respecto del uso de la plataforma? (Los docentes debian ordenar entre cinco opciones empezando por la dificultad máxima).

a. En el manejo técnico de la plataforma (software).

b. En el seguimiento de las actividades de los estudiantes.

c. En la elaboración de las consignas de trabajo.

d. En la elección de la herramienta más adecuada de acuerdo con el tema.

e. En la evaluación de las actividades que realicen los estudiantes.

18. ¿lmaginás alguna otra dificultad no mencionada? ¿Cuál/es? 